\title{
Hypertensive retinopathy and choroidopathy
}

\author{
Wendy Wong MMed, Lekha Gopal DNB, Chee Chew Yip MMed
}

Cite as: CMAJ 2020 April 6;192:E371. doi: 10.1503/cmaj.191275

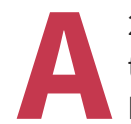

23-year-old man with a 3-year history of essential hypertension presented to the emergency department with blurred vision in his right eye for 2 weeks. His visual acuity was 6/30 in his right eye, and 6/12 in his left eye. Dilated fundus examination showed bilateral malignant hypertensive retinopathy and serous macular detachments (Figure 1). His blood pressure was 222/157 mm Hg. The patient had stopped his antihypertensive medications 1 month previously. There was no secondary cause for his hypertension, or other hypertensive target organ damage. Six weeks after resuming his regular medications, the patient's vision improved to $6 / 9$ in both eyes with regression of the fundus changes.

Signs of hypertensive retinopathy can be detected in $2 \%-14 \%$ of the nondiabetic population aged 40 years and older and it has been identified as a risk marker for stroke and cardiovascular mortality. ${ }^{1}$ Retinal microvascular changes in hypertensive retinopathy, which can be visualized on fundoscopy, are classified as mild, moderate or malignant, using the Mitchell-Wong system. ${ }^{1}$ Generalized retinal arteriolar narrowing and arteriovenous nipping suggest chronic hypertensive damage. More transient effects, reflecting current blood pressure, include focal arteriolar narrowing, retinal hemorrhages, microaneurysms and cottonwool spots. ${ }^{2}$ Malignant hypertension may cause disc swelling due to optic nerve ischemia, and serous macular detachment secondary to fibrinoid necrosis of choroidal arterioles. ${ }^{1}$ Retinopathy findings regress with control of blood pressure. ${ }^{1,2}$ Medication nonadherence is a challenge in managing hypertension and has been shown in a recent meta-analysis to occur in about $45 \%$ of patients. ${ }^{3}$

\section{References}

1. Bhargava M, Ikram MK, Wong TY. How does hypertension affect your eyes? J Hum Hypertens 2012;26:71-83.

2. Wong TY, Mitchell P. The eye in hypertension. Lancet 2007;369:425-35.

3. Abegaz TM, Shehab A, Gebreyohannes EA, et al. Nonadherence to antihypertensive drugs: a systematic review and meta-analysis. Medicine (Baltimore) 2017;96:e5641.
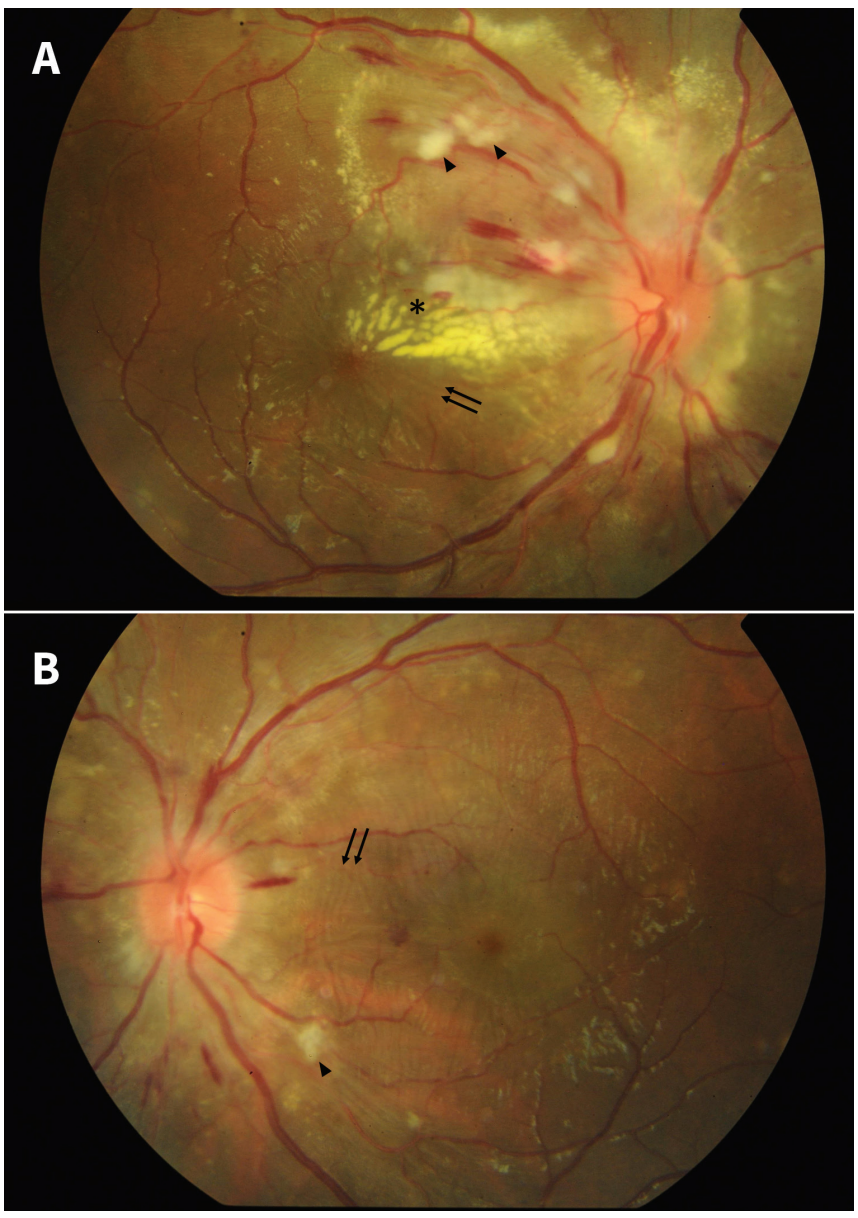

Figure 1: (A) Fundus photo of the right eye of a 23-year-old man, showing disc swelling, splinter hemorrhages, cotton-wool spots along the superotemporal vascular arcade (arrowhead), and hard exudates (asterisk) tracking toward the fovea. There is accumulation of subretinal fluid at the macula (note the fine retinal striae, indicated by the black arrows), which is a feature of hypertensive choroidopathy. (B) Fundus photo of the patient's left eye shows similar but less severe changes.

\section{Competing interests: None declared.}

This article has been peer reviewed.

The authors have obtained patient consent.
Affiliations: Department of Ophthalmology (Wong), National University Hospital; Department of Ophthalmology (Wong, Gopal, Yip), Khoo Teck Puat Hospital, Singapore

Correspondence to: Wendy Wong, Wendy_Wong@nuhs.edu.sg 